\title{
ROBUST MULTIVARIABLE ACTIVE CONTROL WITH SENSORIACTUATOR FEEDTHROUGH
}

\author{
Jeffrey S. Vipperman * \\ Department of Mechanical Engineering \\ 648 Benedum Engineering Hall \\ University of Pittsburgh, Pittsburgh, PA, 15261 \\ David E. Cox ${ }^{\dagger}$ \\ MS 161, Guidance and Controls Branch \\ Flight Dynamics and Controls Division \\ NASA Langley Research Center, Hampton, VA 23681 \\ Robert L. Clark $\ddagger$ \\ Department of Mechanical Engineering and Materials Science \\ Box 90300 \\ Duke University, Durham, NC 27708-0300
}

February 16, 1999

\begin{abstract}
Single and multivariable closed-loop systems using adaptive piezoelectric sensoriactuators are investigated to determine the robust stability characteristics of the plants with respect to the change in feedthrough in the plants. Simulations are performed with an analytical simplysupported plate piezostructure model with surfacemounted piezoceramic bending sensoriactuators. The structured singular value, $(\mu)$, is used in

*Assistant Professor, Member AIAA, ASME

$\dagger$ Graduate Research Assistant at Duke University, Student Member AIAA

${ }^{\ddagger}$ Associate Professor, Member AIAA, ASME

Copyright (C)1999, American Institute of Aeronautics and Astronautics, Inc. All rights reserved.
\end{abstract}

conjunction with an additive uncertainty model to determine the robust stability of the systems with respect to uncertainty in the feedthrough term. Multivariable plants are found to tolerate higher changes in feedthrough, since the offdiagonal transfer paths are unaffected by these errors.

\section{Introduction}

The potential for piezoceramic transducers to simultaneously sense and actuate (sensoriactuator) has been recognized for some time. ${ }^{1,2}$ Any actuation voltage of the piezoceramic shows up as an electrical feedthrough for the piezostructure since the piezoceramic electrically behaves as a capacitor. To complicate matters, this 
feedthrough response is at least two orders of magnitude larger than the charge produced by mechanical strains in the piezoceramic (for 1-D or 2-D structures undergoing bending). Some of the techniques that have been developed to compensate this feedthrough response are handtuned electrical networks, ${ }^{1,2}$ adaptive analog plus digital hybrid methods, ${ }^{3,4}$ and observerbased methods. ${ }^{5}$

The objective of this study is to determine the robust stability characteristics of closed loop structural systems with single or multiple piezoelectric sensoriactuators, as error is introduced into the feedthrough term of the piezoceramic patches. The control system can be designed for any nominal level of piezoelectric feedthrough including no feedthrough (full compensation) and full feedthrough (no compensation). Although different control systems result, the closed loop systems for each case will be identical since the feedthrough is a feedforward portion that can be separated from the feedback portion. Much past effort has been dedicated to ensuring that any drift in the feedthrough due to environmental effects of weak nonlinearities are corrected. ${ }^{3-7}$ This study demonstrates that a relatively large change in feedthrough can be tolerated by the system and still remain stable.

\section{Theory}

\section{Linear Piezoelectric Sensoriactuators}

The linear governing equations of a piezostructure can be written in state space form as:

$$
\begin{aligned}
& \dot{\mathbf{x}}=\mathbf{A x}+\mathbf{B u}+\boldsymbol{\Xi} \mathbf{d} \\
& \mathbf{y}=\mathbf{C x}+\mathbf{D u}+\mathbf{\Upsilon} \mathbf{n}
\end{aligned}
$$

where the signals $\mathbf{x}$ and $\dot{\mathbf{x}}$ are the state vector and its derivative, respectively, $\mathbf{u}$ are the inputs or applied voltages to the piezoelectric sensoriactuators, ${ }^{3} \mathbf{d}$ is a vector of disturbance in$\operatorname{puts}(\mathrm{s}), \mathbf{n}$ is a sensor noise vector, $\mathbf{y}$ is the measured charge outputs of the piezoelectric sensoriactuators. The matrix $\mathbf{A}$ is the dynamics matrix which contains the information about the mass, stiffness and damping of the piezostructure, $\mathbf{B}$ is the input influence matrix for the sensoriactuator voltages and $\boldsymbol{\Xi}$ is the input influence matrix for the exogenous disturbance input(s), $\mathbf{C}$ and $\boldsymbol{\Upsilon}$ are the output influence matrices for the states and sensor noise, respectively, and D is feedthrough matrix, which is ideally the zero matrix. Computation of the A, B, C, and D matrices for a coupled piezostructure model can be found in the literature. ${ }^{8}$ The diagonal feedthrough matrix contains the capacitances of the individual piezoceramic patches as diagonal elements

$$
\mathbf{D}=\left[\begin{array}{cccc}
C_{p, 11} & 0 & \cdots & 0 \\
0 & C_{p, 22} & \cdots & 0 \\
\vdots & \vdots & \ddots & \vdots \\
0 & 0 & \cdots & C_{p, N N}
\end{array}\right]
$$

where $C_{p, N N}$ is the capacitance of the $N^{t h}$ patch. The diagonal nature of matrix $\mathbf{D}$ is consistent with the physical constraint that an applied voltage to one piezoelectric transducer cannot directly cause an electrical response in any other piezoelectric element. This matrix becomes the zero matrix for piezoelectric sensoriactuators having optimally compensated electrical feedthrough. ${ }^{1,2}$ A method of adaptively rejecting the feedthrough response using a blending of analog and digital electronics has been previously presented. ${ }^{3,4}$ Nonidealities in the piezoelectric devices such as hysteresis, leakage, and lossy behavior ${ }^{4,9}$ can preclude ideal or optimal compensation of the capacitive feedthrough matrix, D, resulting in residual diagonal elements. Since the feedthrough response is often orders of magnitude above the mechanical response in a piezoelectric sensoriactuator, a typical phase error of a few tenths of a degree in the compensation network can significantly impact the accuracy of the compensation of the feedthrough. ${ }^{4,9}$

\section{Positivity of Transfer Functions}

For collocated structural control such as occurs using piezoelectric sensoriactuators, the 
transfer function is positive real or minimum The exact form of $\boldsymbol{\Delta}$ is:

phase, implying unconditional closed-loop sta-

bility of direct rate feedback (DRFB) control. ${ }^{10,11}$

It is important to note that the collocated sensoriactuator piezostructure transfer function given by equations (1) and (2) will be positive real regardless of whether the feedthrough matrix is compensated or not. Since the feedthrough term, $\mathbf{D}$, has no phase response and hence is positive real, the superposition of two positive real systems: (A, B, C) and $(\mathbf{D})$ will also be positive real. Thus, the stability guarantees exist with or without compensation of the feedthrough term. ${ }^{12}$

\section{Feedthrough Effects}

The matrix transfer function of a multi-input, multi-output (MIMO) system can be computed from the state space matrices as:

$$
\begin{aligned}
\mathbf{H}(s) & =\mathbf{C}(s \mathbf{I}-\mathbf{A})^{-1} \mathbf{B}+\mathbf{D} \\
\mathbf{H}(s) & =\frac{\mathbf{C} \operatorname{adj}(s \mathbf{I}-\mathbf{A}) \mathbf{B}+\mathbf{D}}{|s \mathbf{I}-\mathbf{A}|}
\end{aligned}
$$

where ()$^{-1}$ represents the matrix inverse, $\operatorname{adj}(\cdot)$ is the matrix adjoint operator and $|\cdot|$ is the matrix determinate. From equation (5) it is easy to see that the zeros of the transfer function are affected by the feedthrough term, D, while the poles of the system are unaffected.

\section{Uncertainty Model}

Equations (2) and (4) show that the effect of the of feedthrough matrix on system model is to add or feed part of the input directly to the system output. In addition, equation (3) shows the diagonal nature of the feedthrough matrix. It is apparent that the uncertainty in the feedthrough model can be represented by an additive block diagonal structure, $\boldsymbol{\Delta}$, as depicted in Figure 1. Note that the additive uncertainty enters the plant through a feedforward path instead of the typical feedback path.

$$
\boldsymbol{\Delta}=\left[\begin{array}{cccc}
\delta+0.1 \delta i & 0 & \cdots & 0 \\
0 & \delta+0.1 \delta i & \cdots & 0 \\
\vdots & \vdots & \ddots & \vdots \\
0 & 0 & \cdots & \delta+0.1 \delta i
\end{array}\right](6)
$$

where $\delta$ represents a real uncertainty which can vary on the interval $[-1,1]$. The term $0.1 \delta i$ represents a small complex portion between $[-0.1 i, 0.1 i]$, which more than adequately represents any possible phase errors in the feedthrough. At the same time, the small complex part greatly improves the numerical properties of the $\mu$ analysis. ${ }^{13}$ Note that the $\mu$ bound does not have a closed form solution and hence involves an iterative technique which produces and upper and lower bound at each frequency. ${ }^{13}$ Slightly more conservative results will be obtained by adding the small complex portion. $\mathcal{H}_{\infty}$ analysis would produce yet more conservative results, since an unstructured (non-diagonal), complex, uncertainty is implicit. Clearly, more accurate robustness results can be obtained by structuring the uncertainty to have good physical agreement with the structure of the piezoelectric feedthrough that is being analyzed, as given by equation (6).

\section{Plant Model}

A plain steel plate measuring $0.60 \times 0.525 \times$ 0.002 meters and having simple supports was modeled. ${ }^{8}$ The disturbance input was a shaker arbitrarily located at normalized coordinates $\{0.3501,0.0960\}$ and corresponding input influence vector, $\boldsymbol{\Xi}$, given by equation (1). The control transducers where piezoelectric sensoriactuators. The simulations used either SISO control with $P Z T_{1}$ listed in Table 1 below, or $4 \mathrm{I} 4 \mathrm{O}$ control with the complete array of patches whose arbitrary center coordinates, which have been normalized with respect to the plate dimensions, are listed in Table 1 . The patches have dimensions of $5.08 \times 5.08 \times 0.002 \mathrm{~cm}$ and are given material properties consistent with 


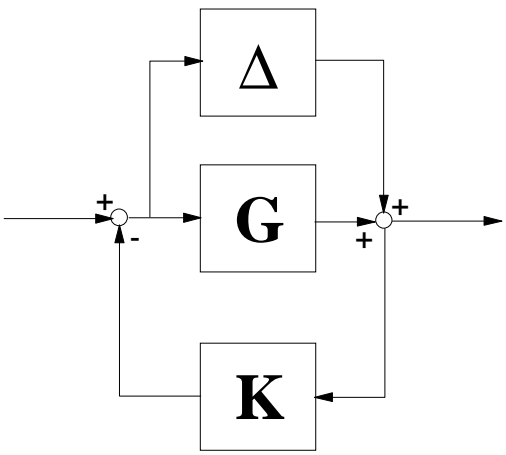

Figure 1: Structured Additive Uncertainty Model Used For Robust Stability Analysis.

the PSI-5A lead zirconate titanate (PZT-5A) material manufactured by Piezo Systems, Inc. The output of each piezoelectric sensoriactuator was low-pass filtered at $300 \mathrm{~Hz}$ with a 4-pole Bessel filter, as would be done in practice. The filter is required to roll off the piezostructure response at higher frequencies, since the nature of the coupling between the piezoelectrics and structure creates a structural compliance that increases with frequency. ${ }^{14}$ The inclusion of the low-pass filter is significant, since it will be shown in the results section that a slightly different $\mu$ bound is achieved when the filters are omitted.

\begin{tabular}{|c|c|c|}
\hline Patch & $x$ coordinate & $y$ coordinate \\
\hline$P Z T_{1}$ & 0.10 & 0.77 \\
$P Z T_{2}$ & 0.82 & 0.41 \\
$P Z T_{3}$ & 0.64 & 0.27 \\
$P Z T_{4}$ & 0.23 & 0.20 \\
\hline
\end{tabular}

Table 1: Normalized Center Locations of PZT Control Sensoriactuators Included in the Simply-Supported Plate Model.

\section{Control Design}

$\mathcal{H}_{2}$ control designs using weightings comparable to linear quadratic Gaussian (LQG) control were computed for all cases. ${ }^{15,16}$ The process and sensor noises are uncorrelated white Gaussian processes. For simplicity, the process noise excites every state of the plate model and likewise the combination of every structural state creates the error variables in the control objective. Unity process noise and error penalty magnitudes were chosen. The sensor noise level was chosen at $10^{-7}$ to be slightly below the pertinent dynamics of the plant in order to achieve good performance while at the same time, creating good roll-off characteristics in the controller. The control effort penalty magnitude was chosen as 5e-6 for the MIMO control system and 5e-7 for the SISO system. An order of magnitude increase in control effort was required for the SISO system in order to achieve performance that was comparable to the MIMO system. A total of 40 modes (80 states) was used in the piezostructure model and consequently the control system contained 96 states (80 structural states and 16 low-pass filter states).

\section{Uncertainty Analysis}

For both the SISO and MIMO systems, the LQG controller was designed, and then a model containing the plant, controller, and uncertainty paths depicted in Figure 1 was created. In lieu of Figure 1, the reader may be more familiar with the " $\mathrm{M}-\boldsymbol{\Delta}$ " structure, where $\mathrm{M}$ is simply the closed-loop plant: $\mathbf{M}=\mathbf{G}(\mathbf{I}+\mathbf{G K})^{-1}$. Estimates of $\mu$ based on $\mathbf{M}$, and $\boldsymbol{\Delta}\left(\mu_{\boldsymbol{\Delta}}(\mathbf{M}(j \omega))\right.$ were then calculated using the $\mu$-Analysis and Synthesis toolbox in Matlab. The actual $\mu$ lies somewhere between the upper and lower bounds that the toolbox returns for $\mu_{\Delta}(\mathbf{M}(j \omega))$. It is the inverse of the maximum $\mu$ which then 
forms the stability margin (SM).

$$
S M=\frac{1}{\max _{\omega} \mu_{\boldsymbol{\Delta}}(\mathbf{M}(j \omega))}
$$

As a comparison for the $\mu$ calculations, the $\mathcal{H}_{\infty}$ bounds were also computed. Since $\mathcal{H}_{\infty}$ represents a more conservative analysis, a lower stability margin is expected, and will provide a measure of the advantage of structuring the uncertainty to fit the problem.

\section{Simulation Results}

\section{Open Loop System}

Figure $2 \mathrm{a}$ and $2 \mathrm{~b}$ depict the open loop singular value plots for the MIMO and SISO control plants, respectively. Two plants are represented in each figure. The solid lines represent plants having full piezoelectric feedthrough and the dotted lines represent plants with no feedthrough. As expected, the feedthrough raises the plant response, particularly between the resonance peaks where the feedthrough response dominates the plant dynamics. In addition, the less dominant plant resonances become hard to distinguish if feedthrough is present (e.g. the first mode at $200 \mathrm{rad} / \mathrm{s}$ ).

Figure 3 shows the sensor response to the process noise used in the LQG design for both the SISO (dotted line) and MIMO (solid line) cases. The constant LQG sensor noise level is also shown in Figure 3 by the horizontal dotdash line. Note that the sensor noise exceeds the response of the first mode for the SISO control plant, since the coupling to the low frequency modes is poor for the given aperture of the PZT transducers. ${ }^{14}$ Consequently, control of that mode is not be expected.

\section{Closed-Loop Results}

Three singular value plots are shown in Figure 4 below. The thin solid line response of the
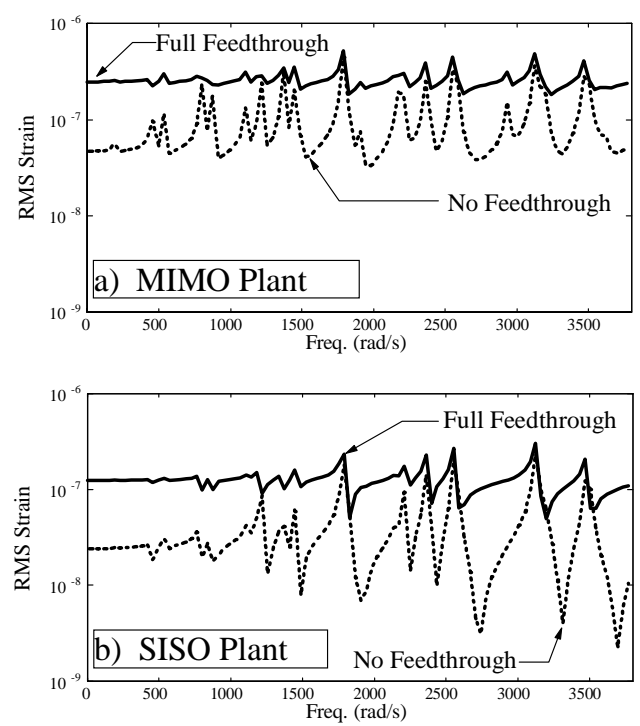

Figure 2: RMS singular value plots for a) MIMO and b) SISO control plants with and without feedthrough.

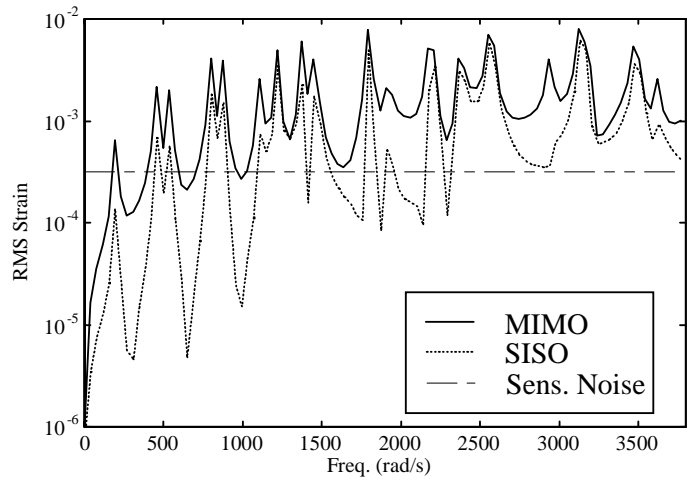

Figure 3: RMS singular value plots of sensor response to process and sensor noises.

open loop error plant. The dotted lines are for the SISO (thin line) and MIMO (thick line) systems. The control systems are moderately aggressive, producing results that are typical of what could be expected in practice. The attenuations of each mode are from no control (at the first mode, $200 \mathrm{rad} / \mathrm{s}$ ) to nearly $18 \mathrm{~dB}$ of attenuation at $1,750 \mathrm{rad} / \mathrm{s}$. 


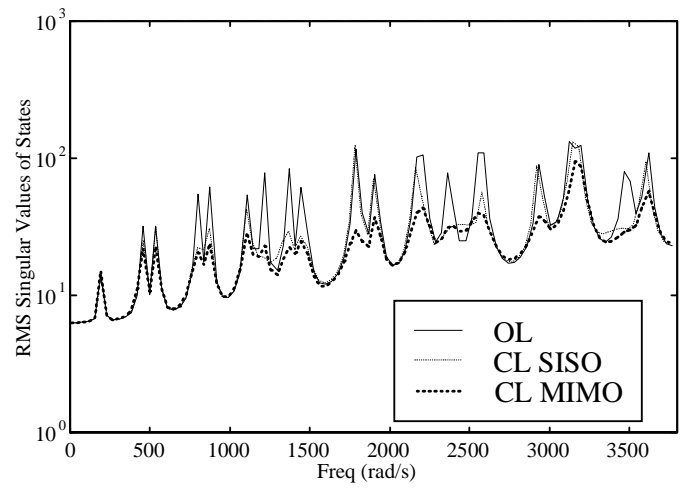

Figure 4: RMS singular value plots of open and closed loop responses for SISO and MIMO systems.

\section{Stability Margins}

As indicated previously, three separate stability margins were computed: the $\mathcal{H}_{\infty}$ bound and the lower and upper $\mu$ bounds. Figure 5 depicts plots of $\mu_{\Delta}^{-1}(\mathbf{M}(j \omega))_{\text {upper }}, \mu_{\Delta}^{-1}(\mathbf{M}(j \omega))_{\text {lower }}$ and $\mathcal{H}_{\infty}^{-1}$ for the SISO system. Since the inverse of $\mu_{\boldsymbol{\Delta}}(\mathbf{M}(j \omega))_{\text {upper }}$ is being plotted, it is the minimum values in Figure 5 with respect to frequency that will form the stability margin. Also, note that these results represent physical units of Farads. Thus, the minimum value in Figure 5 represents the capacitance error in Farads that can be tolerated before instability of the closed loop system results. As expected, the $\mathcal{H}_{\infty}$ result is generally more conservative in the prediction of the stability margin - sometimes by as much as an order of magnitude when compared to the $\mu$ results. The upper and lower $\mu$ bounds in Figure 5 are coalescent, indicating that the actual $\mu$ is known with reasonable accuracy. The minimum value from Figure 5 is $0.0215 \mu \mathrm{F}$ which corresponds to a $21.5 \%$ error in the piezoceramic patch capacitance of $0.1 \mu \mathrm{F}$. To give the reader an idea of the magnitude of temperature change required to create this error in capacitance, the variation in dielectric constant as a function of tem-

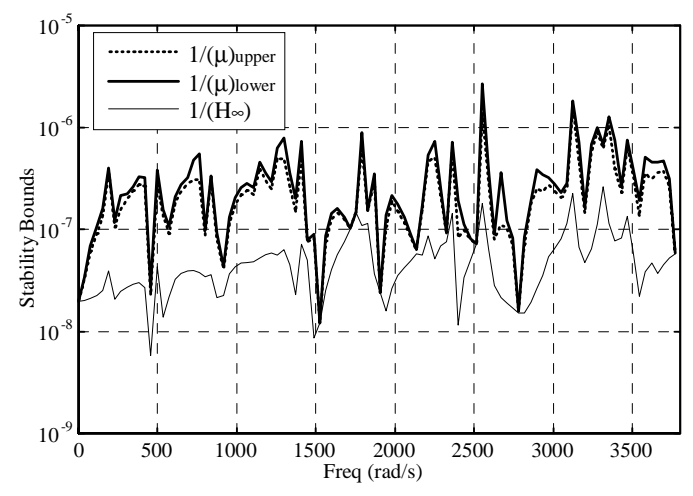

Figure 5: Computed stability bounds for additive uncertainty for SISO system.

perature in the product catalog ${ }^{17}$ was studied. For the PZT-5A material used, it would take a temperature change of $65^{\circ} \mathrm{C}$. In contrast, the more sensitive PZT-5H material would only require a change of $21^{\circ} \mathrm{C}$ in temperature for this change in capacitance to occur. However, for a large group of applications, a 21.5\% in uncompensated capacitance would not be expected.

For the multivariable system using four piezoelectric sensoriactuators, the stability margins are higher. This is expected since the feedthrough matrix is only populated along the diagonal. Thus, the non-driving point paths are unaffected by the capacitance and thus the perturbation in the capacitance. Figure 6 shows the curves for $\mu_{\Delta}^{-1}(\mathbf{M}(j \omega))_{\text {upper }}, \mu_{\Delta}^{-1}(\mathbf{M}(j \omega))_{\text {lower }}$, and $\mathcal{H}_{\infty}^{-1}$ for the MIMO system. The minimum value in Figure 6 is $0.114 \mu \mathrm{F}$, a value that is $114 \%$ of the original capacitance of each patch and more than a factor of five increase from the SISO case. Two other differences between the SISO and MIMO cases can be noted when comparing Figures 5 and 6 . Unlike the SISO case, $\mu_{\boldsymbol{\Delta}}^{-1}(\mathbf{M}(j \omega))_{\text {upper }}, \mu_{\boldsymbol{\Delta}}^{-1}(\mathbf{M}(j \omega))_{\text {lower }}$ are not coalescent for the MIMO case. consequently, it is not known with as much certainty where $\mu$ actually lies, but it will not exceed the upper $\mu$ bound. Surprisingly, in the MIMO case, con- 


\section{Conclusions}

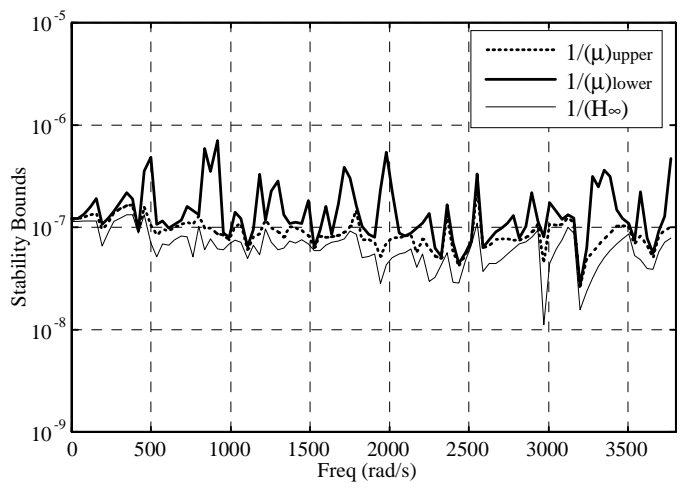

Figure 6: Computed stability bounds for additive uncertainty for MIMO system.

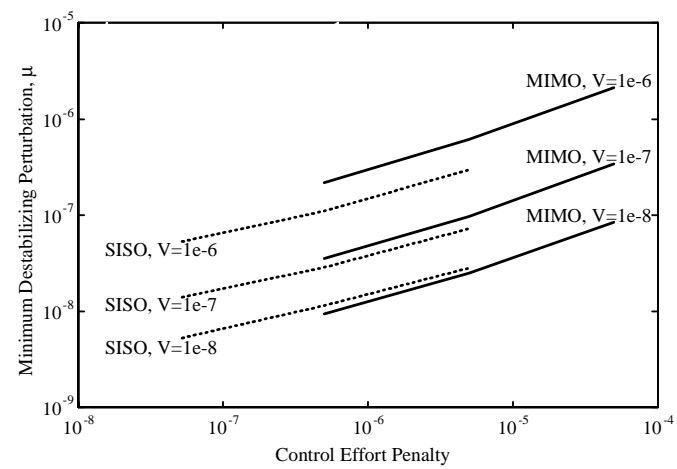

Figure 7: Effect of sensor noise level, $V$, and control effort penalty on minimum perturbation for SISO and MIMO plants.

sideration of the perturbation's structure and real nature didn't significantly improve the prediction of stability margins over $\mathcal{H}_{\infty}$ analysis.

One would expect the stability margins to decrease as the control effort penalty decreases. In a similar manner, one would expect higher stability margins to result from increasing the sensor noise, but at the cost of reducing control performance typically. Indeed these trends are demonstrated in Figure 7, adding a level of confidence to the model and analysis.
A study was conducted in order to determine the sensitivity of piezoelectric sensoriactuators to the amount of feedthrough error. The structured singular value, $\mu$, provided an excellent framework to analyze the effects. An additive, mixed (real + complex), diagonal matrix structure was chosen for the perturbation model, since it physically agrees well with the form of the piezoelectric feedthrough. The system was a simply-supported plate structure having either a single (SISO) or multiple (4I4O) sensoriactuators used as collocated control transducers. An linear quadratic Gaussian control system was developed and the additive uncertainty model appended for the analysis.

It was found that SISO system could tolerate a capacitance error of $21.5 \%$ or $0.0215 \mu$ Farads. This would require a significant temperature fluctuation $\left(65^{\circ} \mathrm{C}\right)$ to create such a change due to thermal drift. For most applications, this would require physical damage to the transducer or the like. The MIMO system was found to be much more robust, being able to tolerate a $114 \%$ change in the original transducer capacitance of $0.1 \mu$ Farads. This is not surprising since the feedthrough and hence the uncertainty only occur for the four driving point paths. For the other 12 paths, there is no feedthrough.

\section{References}

1 Dosch, J. J., Inman, D. J., and Garcia, E., "A self-Sensing Piezoelectric Actuator for Collocated Control," Journal of Intelligent Material Systems and Structures, Vol. 3, Jan. 1992, pp. 166-185.

2 Hagood, N. W. and Anderson, E. H., "Simultaneous Sensing and Actuation Using Piezoelectric Materials," SPIE Vol. 1453, Active and Adaptive Optical Components, San Diego, CA, 1991, pp. 409-421. 
3 Vipperman, J. S. and Clark, R. L., "Implementation of an Adaptive Piezoelectric Sensoriactuator," AIAA Journal, Vol. 34, No. 10, 1996, pp. 2102-2109.

4 Vipperman, J. S., Adaptive Piezoelectric Sensoriactuators for Active Structural Acoustic Control, Ph.D. thesis, Duke University, Durham, NC 27708, 1997.

5 Jones, L. D. and Garcia, E., "Novel Approach to Self-Sensing Actuation," Proceedings of SPIE: Smart Structures and Integrated Systems, San Diego, CA, Vol. 3041, March 3-6 1997, pp. 305-314.

6 Cole, D. G. and Clark, R. L., "Adaptive Compensation of Piezoelectric Sensoriactuators," Journal of Intelligent Material Systems and Structures, Vol. 5, Sep. 1994, pp. 665-672.

7 Spangler, R. L., Broadband Control of Structural Vibration Using Simultaneous Sensing and Actuation With Nonlinear Piezoelectric Ceramics, Ph.D. thesis, M.I.T., Cambridge, MA, Feb. 1992.

8 Hagood, N. W., Chung, W. H., and von Flotow, A., "Modeling of Piezoelectric Actuator dynamics for Active Structural Control," Journal of Intelligent Material Systems and Smart Structures, Vol. 1, No. 3, Jul. 1990, pp. 327-354.

9 Vipperman, J. S. and Clark, R. L., "Complex Adaptive Compensation of Nonlinear Piezoelectric Sensoriactuators," AIAA/ASME Adaptive Structures Forum, Salt Lake City, UT, AIAA-96-1266, April 18-19 1996.

10

Balas, M. J., "Direct Velocity Feedback Control of Large Space Structures," Journal of Guidance and Control, Vol. 2, No. 3, May-Jun. 1979, pp. 252-253.
11 Benhabib, R. J., Iwens, R. P., and Jackson, R. L., "Stability of Large space structure control systems using positivity concepts," Journal of Guidance, Control, and Dynamics, Vol. 4, 1981, pp. 487-494.

12 Cole, D. G. and Robertshaw, H. H., "Direct Rate Feedback for Piezostructures Using Sensoriactuators With Feedthrough Dynamics," AIAA-94-1789-CP, 1994, pp. 514521.

13 Balas, G., Doyle, J., Glover, K., Packard, A., and Smith, R., $\mu$-Analysis and Synthesis Toolbox, MUSYN Inc. and MATHWORKS Inc., Minneapolis, MN 55414-5377, 1995.

14 Vipperman, J. S. and Clark, R. L., "Implications of Using Colocated Strain-Based Transducers for Output Active Structural Acoustic Control," JASA, , 1998, Submitted for Publication.

15 Clark, R. L., Gibbs, G. P., and Saunders, W. R., Adaptive Structures, Dynamics and Control, John Wiley and Sons, New York, 1998.

16 Dorato, P., Abdallah, C., and Cerone, V., Linear-Quadratic Control: An Introduction, Prentice Hall, Englewood Cliffs, NJ 07632, 1995.

17 Piezo Systems, Inc., 186 Massachusetts Ave, Cambridge, MA 02139, Product Catalog. 